\title{
Collaborative Care: Models for Treatment of Patients with Complex Medical-Psychiatric Conditions
}

\author{
Gabriel O. Ivbijaro • Yaccub Enum • Anwar Ali Khan • \\ Simon Sai-Kei Lam • Andrei Gabzdyl \\ Published online: 14 September 2014 \\ (C) The Author(s) 2014. This article is published with open access at Springerlink.com
}

\begin{abstract}
Patients with co-morbidity and multi-morbidity have worse outcomes and greater healthcare needs. Comorbid depression and other long-term conditions present health services with challenges in delivering effective care for patients. We provide some recent evidence from the literature to support the need for collaborative care, illustrated by practical examples of how to deliver a collaborative/integrated care continuum by presenting data collected between 2011 and 2012 from a London Borough clinical improvement programme that compared co-morbid diagnosis of depression and
\end{abstract}

This article is part of the Topical Collection on Complex MedicalPsychiatric Issues

G. O. Ivbijaro

NOVA University, Lisbon, Portugal

G. O. Ivbijaro ( $\square)$

The Wood Street Medical Centre, 6 Linford Road, Walthamstow, London E17 3LA, UK

e-mail: gabriel.ivbijaro@gmail.com

Y. Enum

Public Health Department, Waltham Forest Town Hall, Forest Road, London, UK

e-mail: yaccub.enum@walthamforest.gov.uk

\section{A. A. Khan}

North-East London RCGP Faculty, London, UK

e-mail: anwar.khan@nhs.net

\section{A. A. Khan}

Churchill Medical Centre, Chingford, London, UK

\section{S. S.-K. Lam}

Barking and Dagenham, Havering and Redbridge CCG's, London,

UK

e-mail: simon.lam@onel.nhs.uk

\section{A. Gabzdyl}

Wood Street Medical Centre, Walthamstow, London, UK

e-mail: agabzdyl@hotmail.com other long-term conditions and Accident and Emergency use. We have provided some practical steps for developing collaborative care within primary care and suggest that primary care family practices should adopt closer collaboration with other services in order to improve clinical outcomes and costeffectiveness.

Keywords Collaborative care $\cdot$ Integrated care $\cdot$ Long-term medical conditions (LTC) · Depression · Co-morbidity · Complexity

\section{Introduction}

With a growing population of people living with long-term conditions, many with more than one long-term condition at a time, the answer to managing complexity and multi-morbidity will not come from doing more of the same but from changing the paradigm and finding new ways of working using a person-centred approach [1•], and collaborative care provides such an opportunity.

With the improvements in science, technology and social care as well as better environments, more people are living longer, and the trend towards an ever older population is universal in low-, medium- and high-income countries. In 2011 life expectancy had already exceeded 75 years in 57 countries of the world and, by 2017, the population aged over 65 worldwide will outnumber children under 5 [2]. This change in life expectancy, although welcome, brings its own specific challenges, which include the management of frailty, prevention of social isolation and loneliness, and management of co-morbidity. Complexity and co-morbidity will increasingly become the norm in all regions of the world. Those who commission or provide services for mental health will therefore need to think about new ways of working beyond the traditional boundaries and collaborative care to provide a cost- 
effective method of innovation and achievement of better health especially during the time of recession.

The recent review of projections of global mortality and burden of disease shows that NCDs (non-communicable diseases) and mental illness will continue to be the leading causes of mortality and morbidity in low-medium- and highincome countries [3•] and NCD and mental health comorbidity has an additive effect [4-6]. In a review of 23 low- and medium-income countries, it has been estimated that US\$84 billion of economic production could be lost if nothing is done to address long-term conditions in developing countries [7] and costs to the health system are significant [8••]. This reinforces the need for a collaborative approach to care.

The Global Mental Health Action Plan [9•] and the continuing movement to Universal Health Coverage (UHC) [10] provide a challenge for all actors in health care provision and delivery who strive to deliver evidence-based health care with a good outcome in the face of increasing global morbidity, ageing and the development of new medical technologies within the context of ever dwindling resources, recognising that mental and physical health co-morbidity will continue to be the norm for many and not the exception.

The WHO Mental Health Action Plan 2013-2020 has provided a platform from which all partners can work together towards enabling individuals to achieve full health. This article provides some evidence to support the need for collaborative care and provides some practical examples of how to deliver a collaborative/integrated care continuum.

\section{Collaborative Care Versus Integrated Care}

Social determinants play an important part in health care outcomes and health care delivery, and successful integration/collaboration must go beyond the health sector because people with mental health issues have needs that go beyond health and social care [11]. There is the need to integrate mental and physical health care delivery, social care including housing provision, education, physical health promotion, mental health promotion, mental health advocacy and spirituality across the life course. This will need to be supported by interdisciplinary and multidisciplinary training of the workforce including the creation of a new cadre of family doctors with enhanced skills such as the Primary Care Practitioner with a Special Interest (PwSI) [12].

Although Collaborative Care and Integrated Care are terms that have been used internationally to describe a model of care specifically designed to improve mental health care within a primary care setting, these terms are not used consistently $[13 \bullet, 14 \bullet]$.

Collaborative care is an effective model for integrating behavioural (mental) health care into primary care medical settings. It aims to improve the physical and mental health of people with mental illness. It specifically aims to develop closer working relationships between primary care (family doctors or GPs and practice nurses) and specialist health care (such as Community Mental Health Teams) [15••].

Stroshal [16] defined Collaborative Care as 'Behavioural Health (mental health) working with Primary Care and defined Integrated Care as Behavioural Health (mental health) working within, and as part of, Primary Care Team'.

A functional and practical way to conceptualise the relationship is as a continuum (see Fig. 1). At one end of the continuum there is minimal integration, with mental health services delivered separately from Primary Care (different locations, separate care records and sporadic contact between the agencies). At the other end there is full integration (same team providing both Primary Care and mental health services at the same location with a common care records system).

When this model is applied to many current clinical systems, many primary care practices would be classed as Minimal or Basic collaboration, yet the essence of adopting the Collaborative and Integrated care continuum is to achieve best practice as close to full integration as possible. Full integration, which is difficult to achieve, requires practices to use the same facility, share a common records management system and supporting IT, work in the same team or network, and work toward the same values, philosophy and principles.

Close collaboration/integration will deliver a biopsychosocial model of care by recognising that there is a need to bring together a skill mix to best support the individual experiencing mental illness, recognising that many people with mental illness have other co-morbidities that may be social, psychological, psychiatric and physical [16].

Access to health and social care is often chaotic for people with the complexity and co-morbidity associated with mental illness because there are multiple access points and challenges to overcome [17] before appropriate care can be delivered. To achieve this, services need to be well co-ordinated.

\section{Collaborative Care - The Evidence}

There have been many reviews of collaborative care for the management of patients with chronic illnesses. A randomised controlled trial in 14 primary care clinics in an integrated health care system in Washington State studied patients with depression and poorly controlled diabetes, coronary heart disease or both and concluded that, compared with care as usual, collaborative care involving nurses led to significant improvement in the management of depression and chronic diseases [18•]. In addition to clinical effectiveness, collaborative care has also been demonstrated to be cost-effective [19••].

A meta-analysis of collaborative care for depression and diabetes mellitus noted that patients with diabetes and 
Fig. 1 Conceptualising the collaboration/integration continuum

\begin{tabular}{|c|c|c|c|c|}
\hline MINIMAL & $\begin{array}{c}\text { BASIC } \\
\text { AT A DISTANCE }\end{array}$ & $\begin{array}{l}\text { BASIC } \\
\text { ON-SITE }\end{array}$ & $\begin{array}{c}\text { CLOSE } \\
\text { PARTLY } \\
\text { INTEGRATED }\end{array}$ & $\begin{array}{c}\text { CLOSE } \\
\text { FULLY } \\
\text { INTEGRATED }\end{array}$ \\
\hline & \multicolumn{3}{|c|}{ COLLABORA TION INTEGRA TION CONTINUUM } & \\
\hline $\begin{array}{c}\text { Proximity } \\
\text { Separate sites }\end{array}$ & $\begin{array}{c}\text { Proximity } \\
\text { Separate sites }\end{array}$ & $\begin{array}{l}\text { Proximity } \\
\text { Same facility }\end{array}$ & $\begin{array}{l}\text { Proximity } \\
\text { Same facility }\end{array}$ & $\begin{array}{l}\text { Proximity } \\
\text { Same facility }\end{array}$ \\
\hline Medical records & Medical records & Medical records & Medical records & Medical records \\
\hline $\begin{array}{l}\text { Separate } \\
\text { systems }\end{array}$ & $\begin{array}{l}\text { Separate } \\
\text { systems }\end{array}$ & Separate systems & $\begin{array}{l}\text { Some common } \\
\text { systems }\end{array}$ & $\begin{array}{l}\text { A common } \\
\text { system }\end{array}$ \\
\hline Communication & Communication & Communication & Communication & Communication \\
\hline Sporadic contact & $\begin{array}{l}\text { Communicate } \\
\text { periodically } \\
\text { about shared } \\
\text { patients by } \\
\text { phone or letter }\end{array}$ & $\begin{array}{l}\uparrow \text { communication } \\
\text { due to proximity }\end{array}$ & $\begin{array}{l}\uparrow \text { face to face } \\
\text { communication } \\
\text { due to proximity }\end{array}$ & Same team \\
\hline$\frac{\text { Philosophy \& }}{\underline{\text { Values }}}$ & $\frac{\text { Philosophy \& }}{\text { Values }}$ & $\frac{\text { Philosophy \& }}{\text { Values }}$ & $\frac{\text { Philosophy \& }}{\text { Values }}$ & $\frac{\text { Philosophy \& }}{\underline{\text { Values }}}$ \\
\hline $\begin{array}{l}\text { Separate } \\
\text { cultures }\end{array}$ & $\begin{array}{l}\text { Separate } \\
\text { cultures }\end{array}$ & Separate cultures & $\begin{array}{l}\text { Some shared } \\
\text { culture }\end{array}$ & $\begin{array}{l}\text { Patient } \\
\text { experiences } \\
\text { mental health } \\
\text { treatment as } \\
\text { part of regular } \\
\text { primary care }\end{array}$ \\
\hline
\end{tabular}

(adapted from Milbank [13] \& WHO/Wonca [14]) depression are poorly managed in primary care, which is associated with delayed diagnosis of depression and, once recognised, poor treatment outcomes. Adopting a collaborative approach led to better depression outcomes and improved adherence to treatment for depression and diabetes, suggesting that there is a need to focus on collaborative care that emphasises improvement of the concurrent management of both conditions $[20 \bullet \bullet$.

Another meta-analysis of practice-based interventions for depression concomitant with a range of chronic medical conditions concluded that collaborative care interventions improved outcomes for depression and quality of life in primary care patients with a variety of medical conditions, although the effect was less pronounced in diabetes care $[21 \bullet \bullet]$.

The evidence for the usefulness of collaborative and integrated care for co-morbidity goes beyond common mental disorders, diabetes and coronary heart disease. For example, in respiratory medicine there is an increased risk of depression in COPD (chronic obstructive airways disease) [22], and anxiety also contributes to the use of resources and the cost of COPD management [23-25]. Dealing with COPD alone in primary care, without addressing mental illness co-morbidity, will not lead to the most effective clinical outcomes. Applying the principles of collaborative or integrated care will bring together primary care and respiratory specialists working together to deliver more effective clinical outcomes whilst delivering value for money.

Unützer et al. [26] noted there have been over 70 randomised controlled trials of collaborative care in common mental health disorders that have shown this approach to be more clinically and cost-effective. This suggests that application of the model could substantially improve physical and mental health care in the US Medicare Medicaid system and that Collaborative Care Programmes are one approach to integration and allow primary care providers, care managers and psychiatric consultants to work together to provide better care and monitor patient progress.

There should be integration in health research, policy and practice system-wide, and collaboration must go beyond the health sector. The well-being of the most vulnerable of health system users, whose mental and physical symptoms lead to disorders with persistent impairments, may be a sensitive indicator of a society's need for integrated care and that full 
social participation for vulnerable groups requires sustained access to jobs, schools and other services. This requires cooperation among education, social services, labour and justice sectors [27].

Many successful models of integrated care share common principles. The Wales Health and Wellbeing Best Practice and Innovation Board [28], drawing together some evidence on the determinants of effective integration of health and social care to help inform service re-design, summarised the key determinants as:

- Clarity of strength of purpose - having a shared vision, culture and values that deliver person-centred services based on shared outcome frameworks

- Collaborative leadership at all levels, with expert change management skills and the ability to drive cross-sector working

- A culture of learning and knowledge management that seeks to support the sharing of best practice, improvement and service development across organisational and sector boundaries

- A supportive legislative/policy environment that seeks to create the environment within which integrated services can develop

- Integrated management structures, incorporating the use of joint appointments, with unified leadership and joint governance arrangements and accountability

- Trust-based interpersonal and interprofessional multidisciplinary relationships across sectors, building on the strengths and unique contribution of each partner

- Appropriate resource environments and financial models seeking to ensure collaborative financial models, including the need for pooled budgets

- Comparable information technology (IT) and informationsharing systems that facilitate ease of communication

- Unified performance management systems and common assessment frameworks
- Collaborative capabilities and capacities, with all practitioners being skilled in integrated working and management

England has been making efforts to scale up integrated/ collaborative care. A recent report for the Department of Health in England covering 16 Integrated Care Pilots (ICPs) [29••], some of which specifically included some mental health and dementia services, concluded that where there had been perceived benefits, facilitators to ICP success included strong leadership and pre-existing relationships at a personal level across organisations, shared values, collective communicated vision, investment of effort in widespread staff engagement and the provision of education and training specific to service change.

Large-scale, complex integrations were a barrier to success, as were staff concerns about changes to their roles or even threat to their jobs and poor IT connectivity between systems and organisations in a holistic fashion.

This is consistent with the findings of a review by the Kings Fund in the UK [8••]. An example from New Zealand also follows the same principles to achieve success in the delivery of integrated care [30].

One of the tools that can be used to integrate across sectors and services, especially for those with long-term health conditions with multi-morbidity who may also be vulnerable, is through the use of Navigators or care co-ordinators. In the past most care co-ordinators have been nurses or other clinicians. Non-clinicians such as Navigators can also be effective in supporting improved integration/ collaboration.

A Navigator is a single named individual who can help people navigate their way through complex systems across health, social care, housing, employment and education (among other services) and help to pull together integrated care packages $[31,32]$. This would go a long way to ensuring that people received effective integrated care.
Fig. 2 Asthma, depression, A\&E admissions and cost in Waltham Forest 2011-2012
Emergency Admissions of patients with Asthma vs Depression \& Asthma

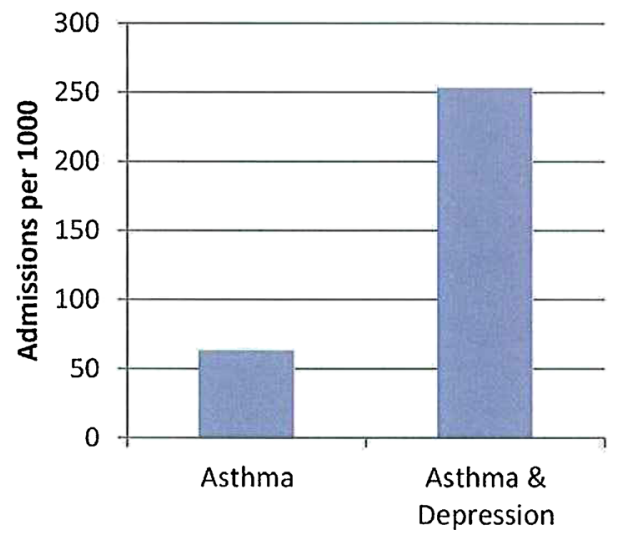

Cost comparison between patients with Asthma vs Depression \& Asthma

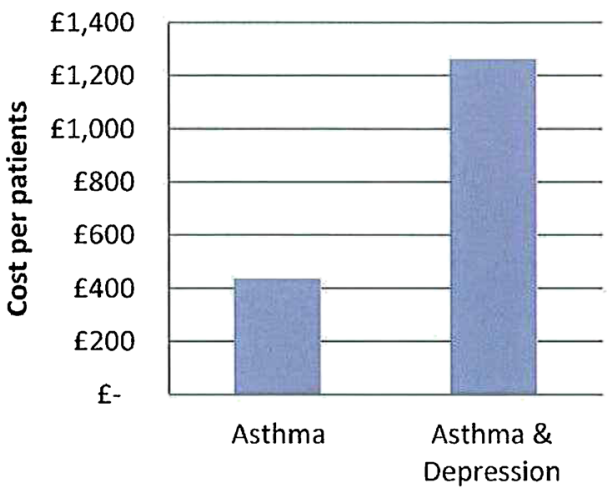


Waltham Forest Commissioning Consortia Regression Analysis

Depression Vs. Asthma

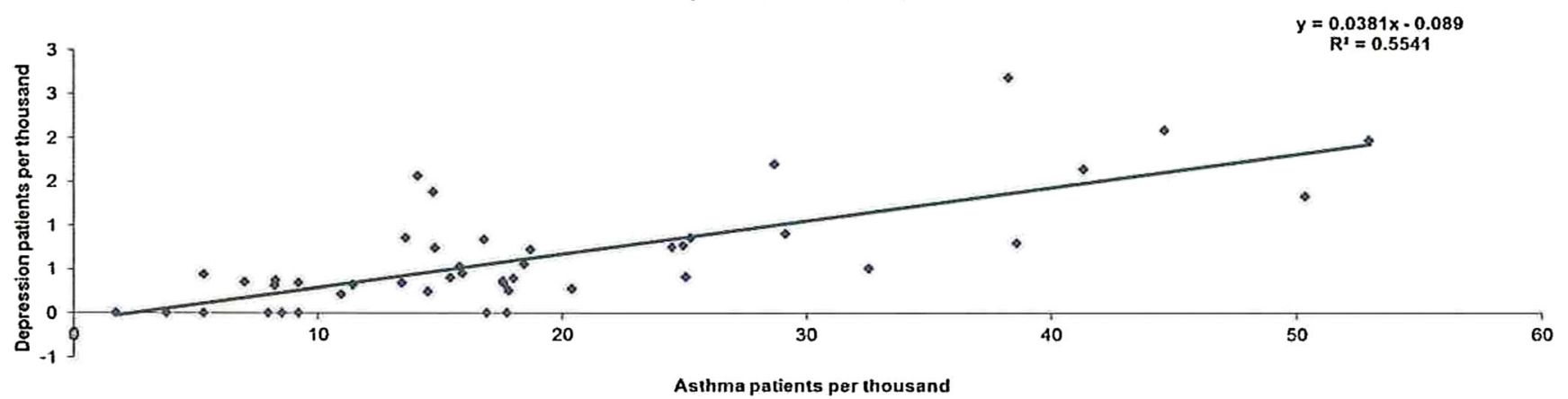

Fig. 3 Prevalence of asthma and depression per Waltham Forest GP practice 2011-2012

\section{Case Example - Cost and Co-Morbidity, the Experience of Waltham Forest, London}

Waltham Forest is a Borough in East London with a diverse population of 258,249 comprising $42 \%$ Black and Asian minority ethnicities. It is $51 \%$ female with an elderly population in the north of the borough and younger population in the south. There is a high birth rate, a high prevalence of lowbirth-weight babies and a relatively young population compared to England, and it is above the national average in the 010 and 20-44 age groups; the older population is projected to grow. Waltham Forest is the 15th most deprived borough in England, levels of deprivation are increasing over time, and $33 \%$ of households are defined as income deprived and $20 \%$ of households have no member in employment.

As part of Waltham Forest's initiative to reduce costs and improve clinical outcomes a software package called Health Analytics was developed to capture patient data in general practice and secondary care. The data collected using Health Analytics specifically captured Accident and Emergency attendance, in-patient hospital admissions, out-patient attendance, general practice appointments and diagnosis of long- term health conditions. These data were then used to calculate the cost of care per 1,000 patients registered at each practice and aggregated for all individual general practice providers in Waltham Forest taking into account the total number of A\&E attendance per year and their cost, the number of A\&E admissions per year and their cost, the number of short stay admissions ( $<3$ days) and their cost, and the number of longer stay admissions ( $>3$ days) and their cost. The data were reviewed to test whether there was a relationship between the prevalence of depression and the prevalence of other long-term health conditions in the Waltham Forest general practice population.

The data presented in this case study are for a total Waltham Forest patient population of 258,249 as registered in 20112012. Figures 2, 3, 4, 5, 6, 7, 8, 9, 10, 11, 12, and 13 show that when depression is co-morbid with other long-term health conditions, the cost of treatment is significantly increased and is also associated with a disproportionate use of accident and emergency care. This is true for all the other long-term conditions evaluated. For example, the average cost per patient with asthma and depression is almost three fold compared to the cost per patient of asthma alone. The points on
Fig. 4 Coronary heart disease (CHD), depression, A\&E admissions and cost in Waltham Forest 2011-2012
Emergency Admissions of patients with CHD vs Depression \& CHD

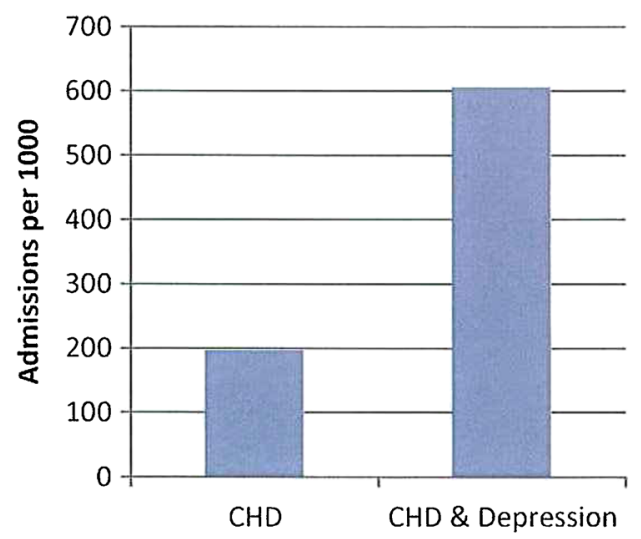

Cost Comparison between patients with CHD vs Depression \& CHD

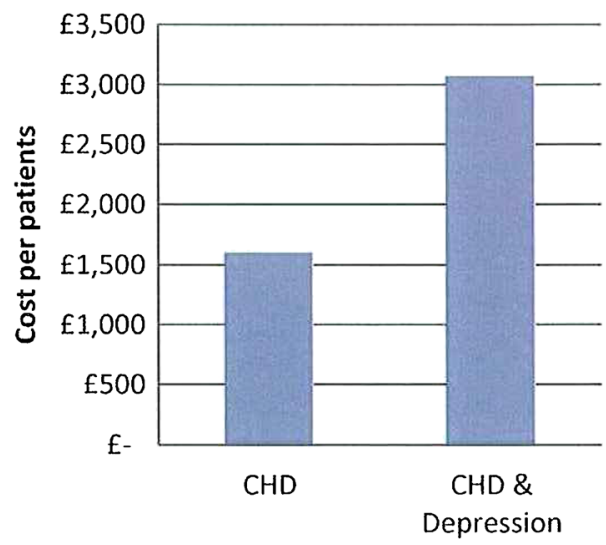




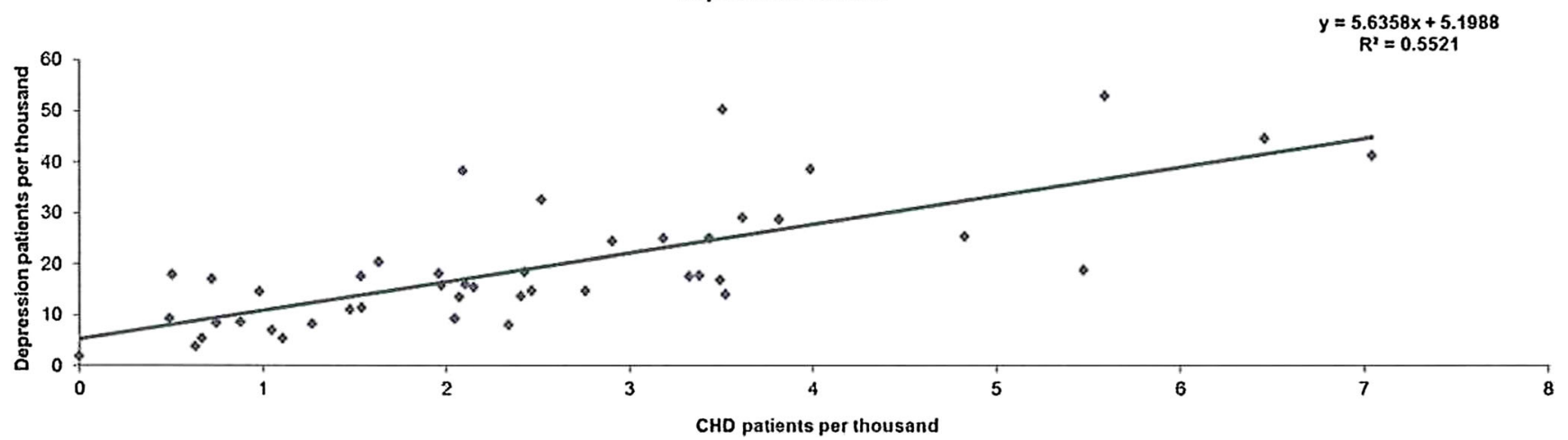

Fig. 5 Prevalence of coronary heart disease (CHD) and depression per Waltham Forest GP practice 2011-2012

each correlation represent a general practice service and for each condition the correlation graphs show that practices with high prevalence of depression also have high prevalence of depression. In Waltham Forest the association is strongest for heart failure and hypertension.

The left side of Fig. 2 compares the A\&E admission rates for patients with a diagnosis of asthma alone and those with a diagnosis of asthma co-morbid with depression. The right side of Fig. 2 compares the average cost per patient for those with a diagnosis of asthma alone (£437.00) and those with a diagnosis of asthma co-morbid with depression (£1,263.00).

The points on each correlation in Fig. 3 represent a general practice service and the correlation graph shows that practices with high prevalence of depression also have high prevalence of asthma.

The left side of Fig. 4 compares the A\&E admission rates for patients with a diagnosis of coronary heart disease (CHD) alone and those with a diagnosis of coronary heart disease (CHD) co-morbid with depression. The right side of Fig. 4 compares the average cost per patient for those with a diagnosis of coronary heart disease (CHD) alone $(£ 1,603.00)$ and those with a diagnosis of coronary heart disease (CHD) co-morbid with depression ( $£ 3,072.00)$.

The points on each correlation in Fig. 5 represent a general practice service and the correlation graph shows that practices with high prevalence of depression also have high prevalence of coronary heart disease (CHD).

The left side of Fig. 6 compares the A\&E admission rates for patients with a diagnosis of cancer alone and those with a diagnosis of cancer with depression. The right side of Fig. 6 compares the average cost per patient for those with a diagnosis of cancer alone $(£ 1,950.00)$ and those with a diagnosis of cancer co-morbid with depression (£2,786.00).

The points on each correlation in Fig. 7 represent a general practice service and the correlation graph shows that practices with high prevalence of depression also have high prevalence of cancer.

The left side of Fig. 8 compares the A\&E admission rates for patients with a diagnosis of diabetes alone and those with a diagnosis of diabetes co-morbid with depression. The right
Fig. 6 Cancer, depression, A\&E admissions and cost in Waltham Forest 2011-2012
Emergency Admissions of patients with Cancer vs Depression \& Cancer

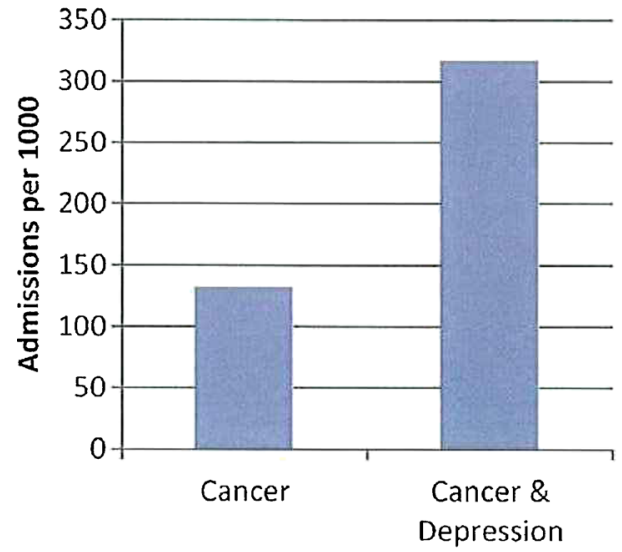

Cost comparison between patients Cancer vs Depression \& Cancer

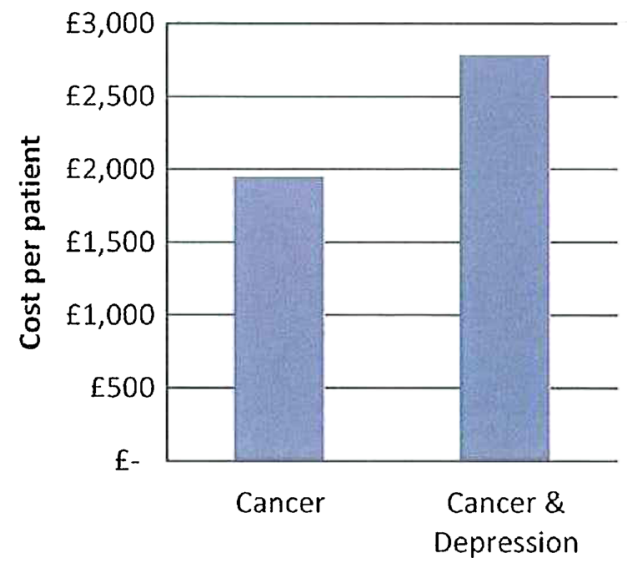


Waltham Forest Commissioning Consortia Regression Analysis

Depression Vs. Cancer

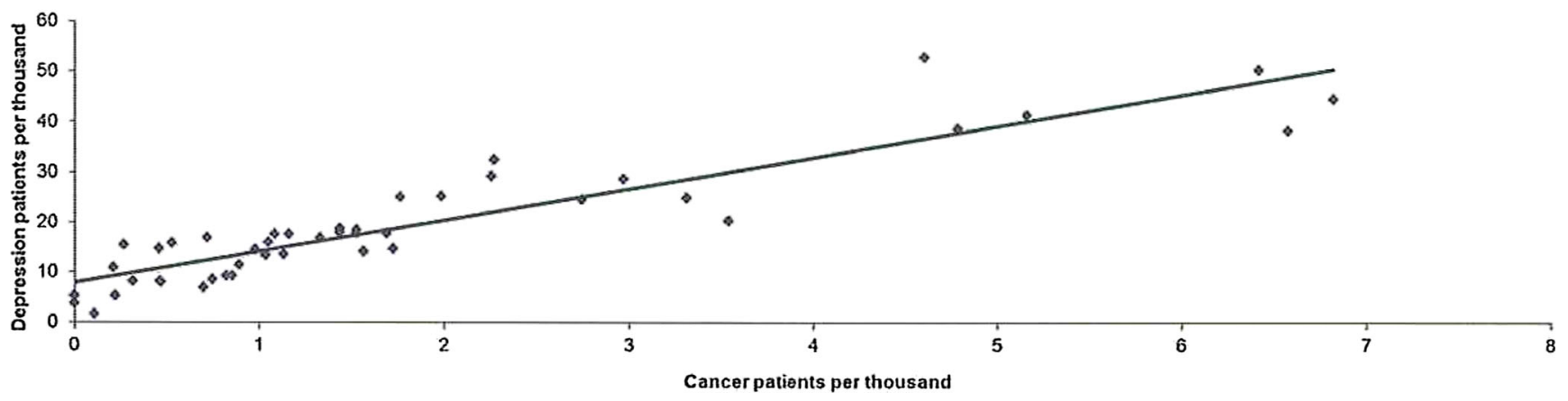

Fig. 7 Prevalence of cancer and depression per Waltham Forest GP practice 2011-2012

side of Fig. 8 compares the average cost per patient for those with a diagnosis of diabetes alone $(£ 650.00)$ and those with a diagnosis of diabetes co-morbid with depression $(£ 1,786.00)$.

The points on each correlation in Fig. 9 represent a general practice service and the correlation graph shows that practices with high prevalence of depression also have high prevalence of diabetes.

The left side of Fig. 10 compares the A\&E admission rates for patients with a diagnosis of heart failure alone and those with a diagnosis of heart failure co-morbid with depression. The right side of Fig. 10 compares the average cost per patient for those with a diagnosis of heart failure alone $(£ 2,646.00)$ and those with a diagnosis of heart failure co-morbid with depression $(£ 4,460.00)$.

The points on each correlation in Fig. 11 represent a general practice service and the correlation graph shows that practices with high prevalence of depression also have high prevalence of heart failure.

The left side of Fig. 12 compares the A\&E admission rates for patients with a diagnosis of hypertension alone and those with a diagnosis of hypertension co-morbid with depression.
The right side of Fig. 12 compares the average cost per patient for those with a diagnosis of hypertension alone (£741.00) and those with a diagnosis of hypertension co-morbid with depression $(£ 1,607.00)$.

The points on each correlation in Fig. 13 represent a general practice service and the correlation graph shows that practices with high prevalence of depression also have high prevalence of hypertension.

Scheduled care is less costly and more cost-effective in primary care and secondary care. Waltham Forest patients with long-term conditions co-morbid with depression showed increased use of episodic care. This case example from the London Borough of Waltham Forest illustrates the need for a collaborative approach for dealing with co-morbid long-term conditions. Depression increases the cost of the management of other long-term health conditions and consistent with the findings in the literature [4-6] effective management of both the depression and the long-term physical condition is necessary to achieve the best outcomes.

Waltham Forest is currently evaluating a range of pilots that have been initiated in response to our findings. These pilots
Fig. 8 Diabetes, depression, A\&E admissions and cost in Waltham Forest 2011-2012

\section{Emergency Admissions of patients with Diabetes vs Diabetes \& Depression}

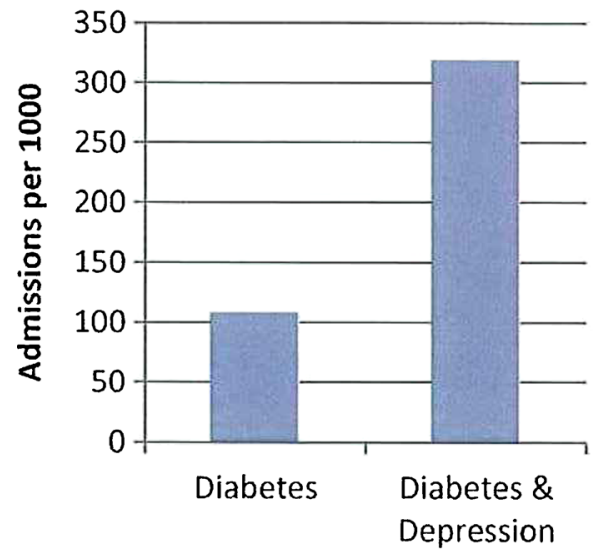

Cost comparison between patients with Diabetes vs Depression \& Diabetes

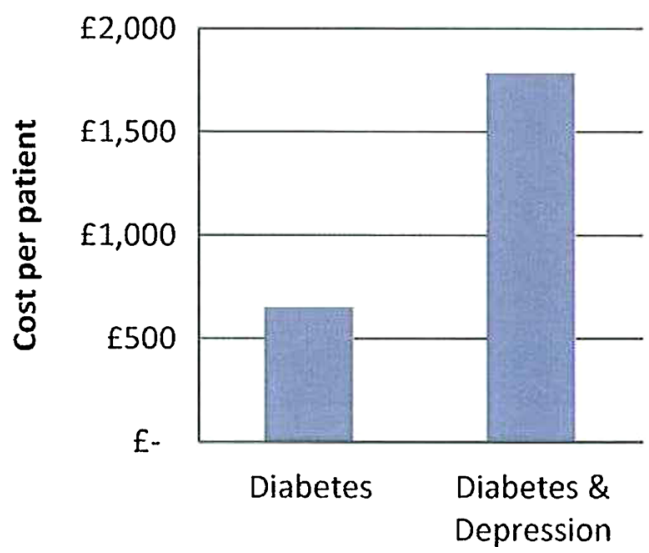


Waltham Forest Commissioning Consortia Regression Analysis

Depression Vs. Diabetes

$R^{x}=0.5642$

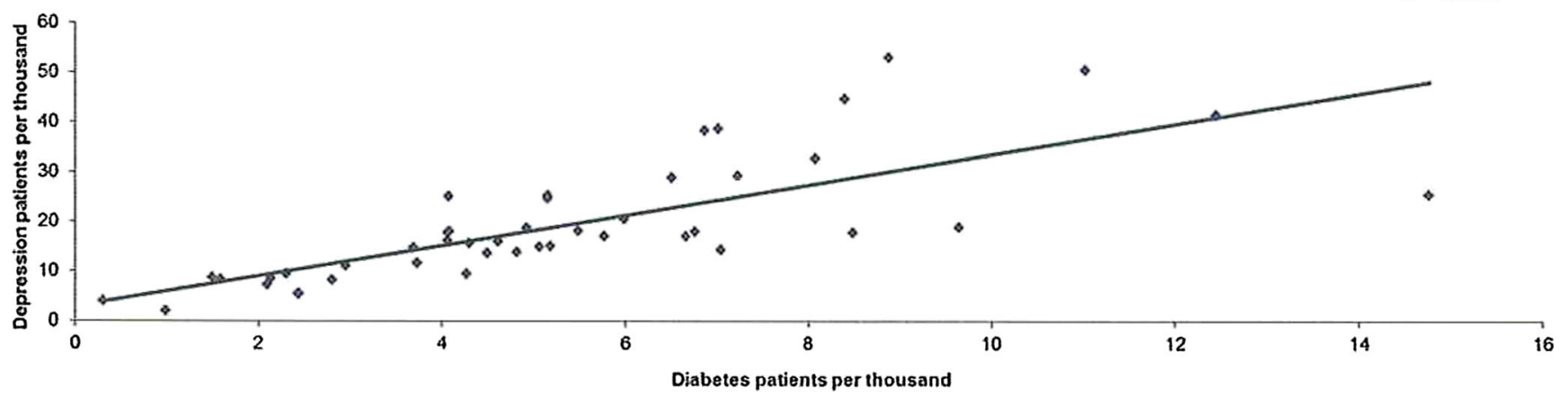

Fig. 9 Prevalence of diabetes and depression per Waltham Forest GP practice 2011-2012

have highlighted the need to improve the sharing of data and information between health and social care professionals and learning together to enable better understanding of each other's work streams.

\section{Developing Your Integrated Service}

One of the aims of this article is to help individual practices and practitioners to implement or accelerate their collaborative/integrated approach to patient care. In order to develop an integrated/collaborative care service to suit your local population, you will first need to understand the population you are serving and the existing pathways to care. This will include an understanding of the determinants of health, the legal framework in which you practice, the range of third sector or non-government organisations available in your area and the provision delivered by existing secondary care providers. (See Fig. 14) [38].
There will need to be a business model and a project plan with clear time lines including the skill mix and workforce necessary (such as doctors, nurses, psychologists, health care assistants, etc.) to deliver the model. It is important to recognise that some of the tasks currently undertaken by clinicians can be done by others of varying grades. For example, rather than trained nurses, navigators may be able to deliver care coordination. You have to deal with physical and mental health conditions together, as part of a stepped care approach [33•, 34-38].

Collaborative/integrated care requires accountability from all the organisations involved in the partnership or collaboration. It requires clear clinical leadership and a methodology for information sharing. It is important to support this through a clear methodology of payments or incentives that are clearly established and understood before the project begins. This forms the foundation for effective collaboration/integration.

There are some specific tasks that need to take place in primary care. These will include training staff in patient presentation, assessment and diagnosis. Once diagnosis is made it
Fig. 10 Heart failure (HF), depression, A\&E admissions and cost in Waltham Forest 20112012

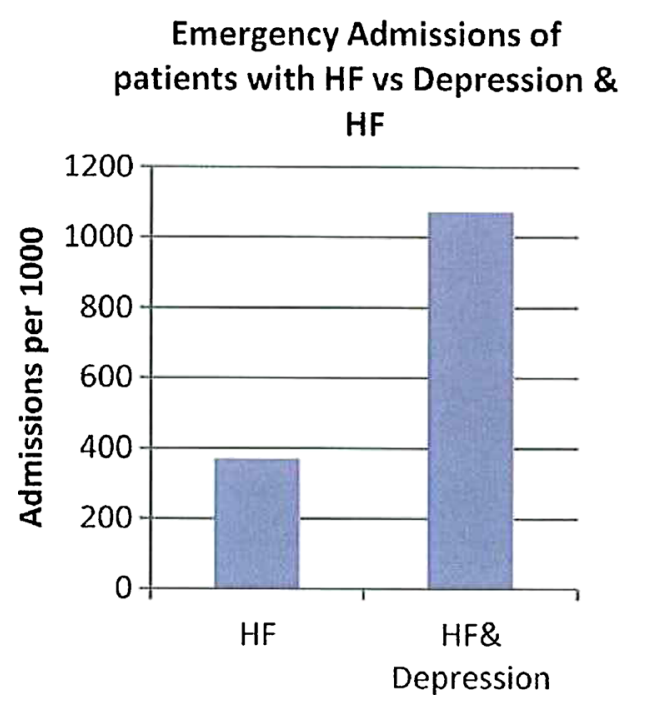

Emergency Admissions of $\mathrm{HF}$

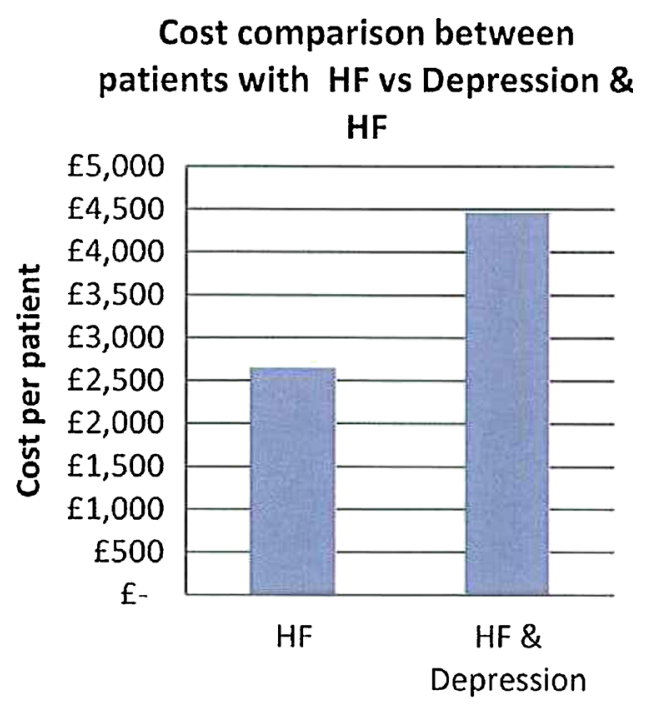


Waltham Forest Commissioning Consortia Regression Analysis

Depression Vs. Heart Failure

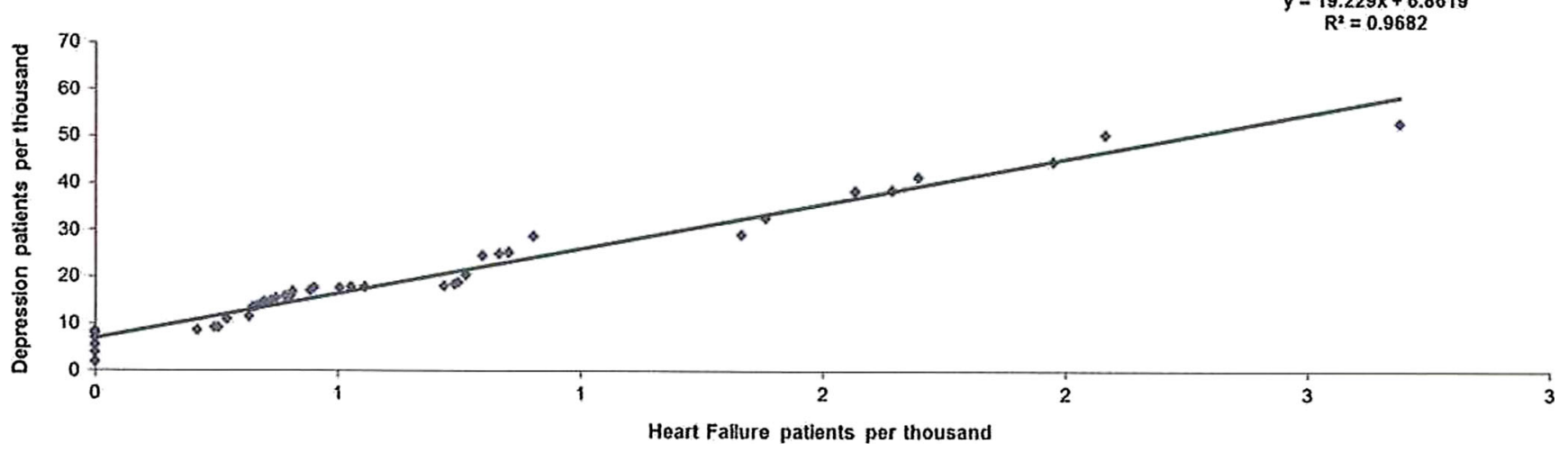

Fig. 11 Prevalence of heart failure (HF) and depression per Waltham Forest GP practice 2011-2012

should be entered onto the practice disease register. There is also a need to train staff to stratify patients based on the risk and seriousness of their condition so that they can be directed to the right level of resources as early as possible.

Primary care interventions need to be supported by an agreed clinical protocol and guideline, and it is often better to adopt a stepped care approach. Clinical protocols and guidelines should also include a clear statement of when to refer to secondary care and when secondary care should discharge back to primary care.

It is essential that all partners agree to the clinical protocols and guidelines for each condition so that they are universally adopted across the collaboration, underpinned by good record keeping and information that can be shared between partners. Patients, their families and carers should be part of the decision-making, and there should be continuous evaluation of outcomes so that services can be re-designed whenever the local population needs change.
Good collaborative/integrated care must have cognisance of community and personal resilience, including self-care, and should universally promote smoking cessation as we now know that smoking cessation is associated with a reduction in depression, anxiety and stress and associated with improved mood and quality of life [39••]. This is an activity that can take place across the collaborative, be it community, primary or secondary care.

\section{Conclusion}

The management of complexity and multi-morbidity, especially of long-term conditions and depression, will continue to pose challenges for those who commission health services and for those who deliver clinical interventions. Primary care will play an increasingly significant role with the move to universal health coverage. There is a need to develop new ways of
Fig. 12 Hypertension, depression, A\&E admissions and cost in Waltham Forest 20112012

\section{Emergency Admissions of patients with Hypertension \& Depression}

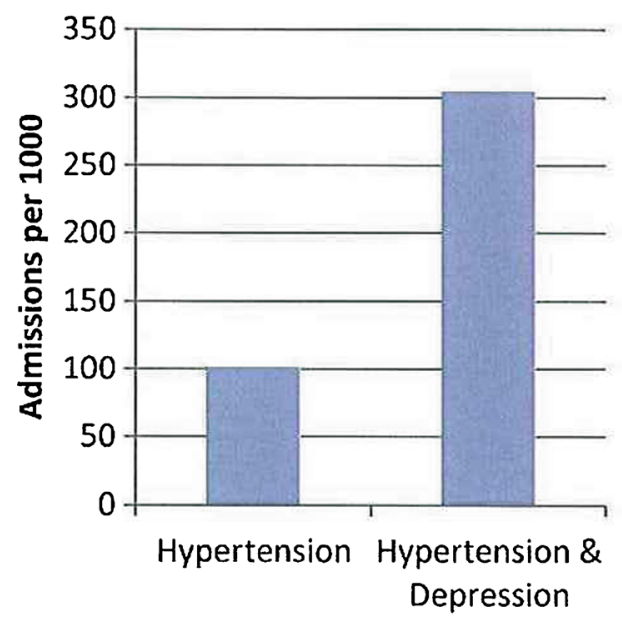

Cost comparison between patients with Hypertension vs Depression \& Hypertension

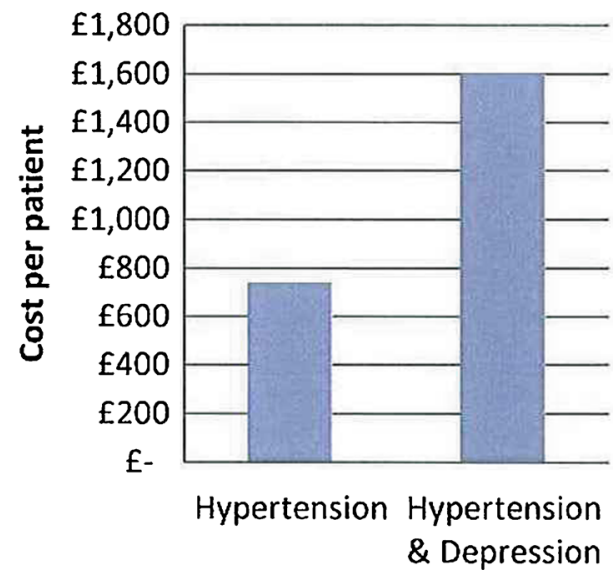


Waltham Forest Commissioning Consortia Regression Analysis

Depression Vs. Hypertension

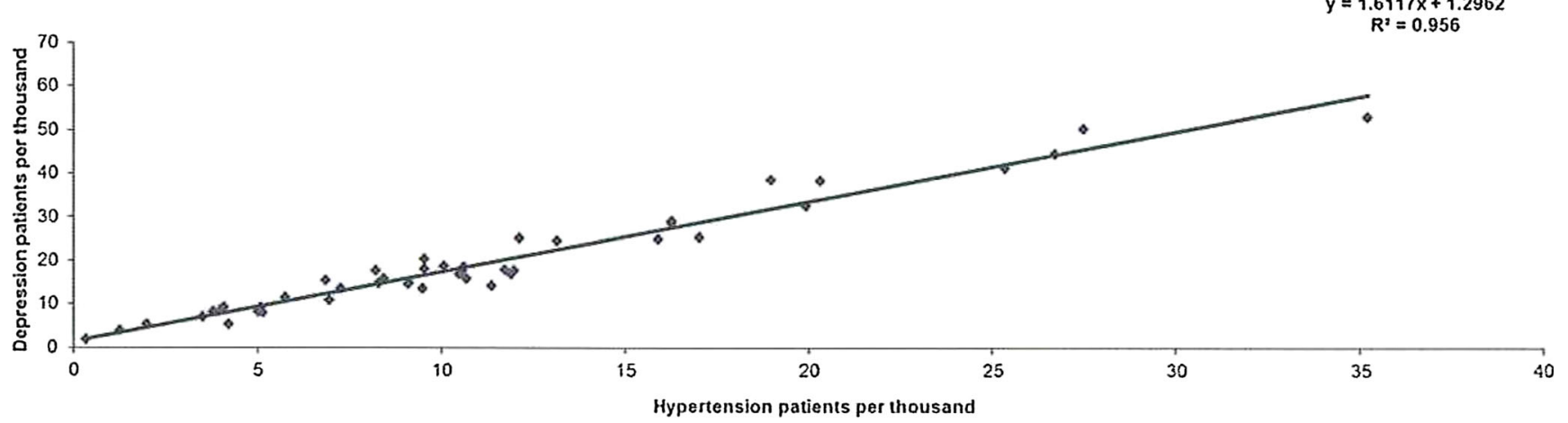

Fig. 13 Prevalence of hypertension and depression per Waltham Forest GP practice 2011-2012

delivering clinical care to a diverse, ageing population with increasingly complex needs arising from the multi-morbidity associated with mental illness, especially depressive disorder.

This review has brought together the current best evidence supporting the collaborative care model in primary care. We have shown that when long-term medical conditions are comorbid with depression clinical outcomes worsen and economic costs increase.
There is a growing evidence base supporting a collaborative approach to the delivery of clinical care because this is associated with better individual and community health outcomes and is also economically efficient. We have put forward a schema to illustrate how a collaborative system may be put into practice.

There is evidence that collaborative care is clinically and cost-effective in the management of care of patients with co-
Fig. 14 Collaborative mental health care - a schema

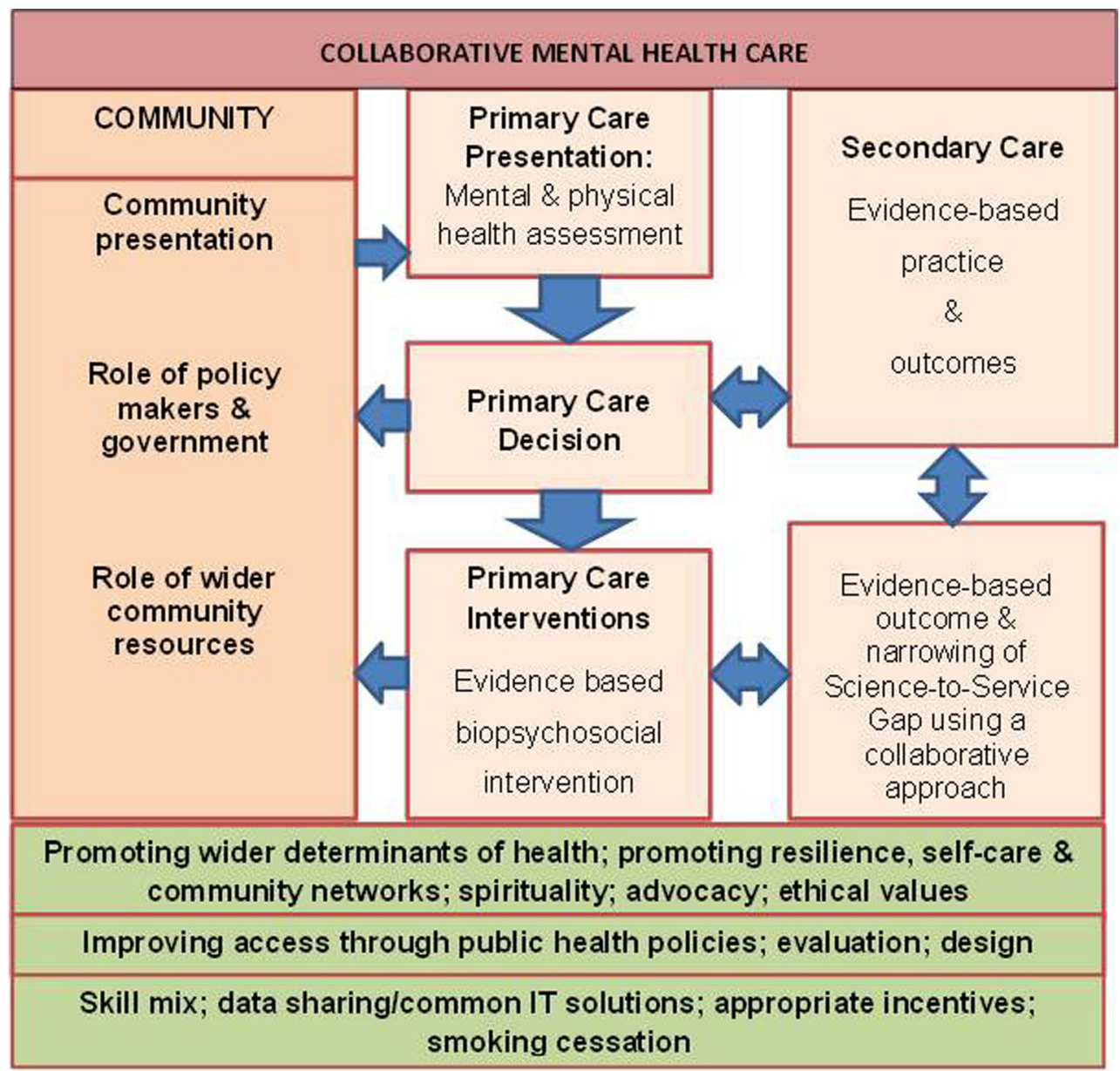

(Adapted from Ivbijaro 2012 with permission[38]) 
morbid depression and other long-term conditions. Primary care family practices should adopt closer collaboration with other services in order to improve clinical outcomes. The case example from the London Borough of Waltham Forest shows that there is a need to invest not just in physical health care but also in mental health care because the prevalence of depression increases as the prevalence of other long-term conditions increases as there is a disproportionate rise in cost when managing comorbid mental and physical health conditions.

The literature evidence illustrates that the experience in the London Borough of Waltham Forest is not unique so there is a need to encourage innovation through collaborative care because it affords the opportunity to manage co-morbidity and multimorbidity in a cost-effective way.

Acknowledgments We are grateful to the Health Analytics Team in Barking and Dagenham, Havering and Redbridge CCGs, the London Borough of Waltham Forest Public Health Department and Waltham Forest CCG and to Professor Rachel Jenkins, Professor Todd M. Edwards, Professor Sir David Goldberg, Dr Lucja Kolkiewicz and Professor Michelle Riba for their comments on earlier drafts of this paper.

\section{Compliance with Ethics Guidelines}

Conflict of Interest Gabriel O. Ivbijaro, Yaccub Enum, Anwar Ali Khan, Simon Sai-Kei Lam, and Andrei Gabzdyl declare that they have no conflict of interest.

Human and Animal Rights and Informed Consent This article does not contain any studies with human or animal subjects performed by any of the authors.

Open Access This article is distributed under the terms of the Creative Commons Attribution License which permits any use, distribution, and reproduction in any medium, provided the original author(s) and the source are credited.

\section{References}

Papers of particular interest, published recently, have been highlighted as:

- Of importance

.. Of major importance

1. Reeve J, Blakeman T, Freeman GK, Green LA, James PA, Lucassen P, et al. Generalist solutions to complex problems: generating practice-based evidence-the example of managing multimorbidity. BMC family Practice. 2013;14:112-20. This article addresses the need to innovate when managing complexity by providing a novel framework for conceptualising complexity whilst keeping the patient at the centre of care.

2. World Health Organisation. Health statistics and health information systems. http://apps.who.int/ghodata/?vid=720. Accessed 7 April 2013.

3. Mathers CD, Loncar D. Projections of global mortality and burden of disease from 2002 to 2030. PLoS Med. 2006;3(11):2011-29. This paper brings the widely quoted 1996 Murray and Lopez paper on global and regional projections of mortality and burden of disease by cause for the years 2000, 2010 and 2030 up to date. It enables those who commission services to be able to better plan for the future and quantifies the growing influence of NCD's (noncommunicable disease) on mortality and morbidity.

4. Katon WJ, Von Korff M, Lin EHB, Simon G, Ludman E, Russo J, et al. The pathways study. A randomized trial of collaborative car in patients with diabetes and depression. Arch Gen Psychiatry. 2004;61:1042-9.

5. Ivbijaro G. Mental health: a resilience factor against both NCDs and CDs. In: Commonwealth Health Partnerships 2012. Cambridge: Nexus Strategic Partnerships, 2012, pp. 17-20.

6. World Health Organization. The global burden of disease: 2004 update. Geneva: The World Health Organization; 2008.

7. Abegunde DO, Mathers CD, Adam T, Ortegon M, Strong K. The burden and costs of chronic diseases in low-income and middleincome countries. Lancet. 2007;370(9603):1929-38.

8.• Naylor C, Parsonage M, McDaid D, Knapp M, Fossey M, Galea A. Long-term conditions and mental health. The cost of co-morbidities. London: The King's Fund and Centre for Mental Health; 2012. This excellent review highlights the role of mental health in contributing to the cost of care for other co-morbid long-term conditions. It addresses detection and integration and provides a range of practical examples illustrating successful integrated/ collaborative care.

9. World Health Organization. Mental health action plan 2013-2020. Geneva: The World Health Organization; 2013. This document was produced in response to the World Health Assembly Resolutions WHA66.8 and WHA65.4, provides the necessary framework for improving mental health globally from the government to individual level, and provides a strong rationale for collaborative care.

10. World Health Organization. The world health report - health system financing: the path to universal coverage. Geneva: The World Health Organization, 2010 http://www.who.int/whr/2010/en/.

11. Marmot M. Fair society, healthy lives: the marmot review. UCL Institute of health Equity:2010.

12. Department of Health, Royal College of General Practitioners, Royal Pharmaceutical Society of Great Britain, NHS Primary Care Contracting. Guidance and Competences for the Provision of Services Using Practitioners with Special Interest (PwSI's) Mental Health. London: 2009

13. Collins C, Hewson DL, Munger R, Wade T. Evolving models of behavioural health integration in primary care. New York: Milbank Memorial Fund; 2010. This very good review addresses some of the misunderstandings arising from the use of different terminologies for collaborative/integrated care. It provides a historical perspective for collaborative care and provides researchers and clinicians from across the globe with a common framework.

14. WHO/Wonca. Integrating mental health into primary care: a global perspective. Geneva: WHO; 2008. This document was jointly produced by WHO and Wonca to bring together the evidence that primary care can deliver effective mental health care in a nonstigmatising environment using a collaborative approach that values self-care.

15.• Reilly S, Planner C, Gask L, Hann M, Knowles S, Druss B, Lester H. Collaborative care approaches for people with severe mental illness. Cochrane Database Syst Rev 2013, Issue 11. Art. No.: CD009531. doi:10.1002/14651858.CD009531.pub2. See more at: http://summaries.cochrane.org/CD009531/SCHIZ_collaborativecare-approaches-for-people-with-severe-mental-illness $\% 23$ sthash. $2 \mathrm{WjAw} Y x u$.dpuf. This excellent systematic review brings us up to date with the evidence for delivering collaborative care to those people with severe mental illness.

16. Strosahl K. Integrating behavioural health and primary care services: the primary mental health care model. In: Blount A, editor. Integrated primary care: the future of medical and mental health collaboration. New York: W.W. Norton; 1998. p. 139-66. 
17. WHO. Integrating mental health into primary care. Mental Health Policy, Planning and Service Development Information Sheet 3. WHO Geneva: 2007.

18. Katon W, Lin EHB, Von Korff M, Ciechanowski P, Ludman EJ, Young B, et al. Collaborative care for patients with depression and chronic illnesses. N Engl J Med. 2010;363(27):2611-20. This single-blind randomised controlled trial in 14 primary care clinics in an integrated health care system in Washington State, USA, shows that collaborative care was superior to usual care in the management of depression and other chronic illnesses and illustrates the effectiveness of nurses in this role.

19.• Katon W, Russo J, Lin EHB, Schmittdiel J, Ciechanowski P, Ludman E, et al. Cost-effectiveness of a multicondition collaborative care intervention. Arch Gen Psychiatry. 2012;69(5):506-14. This randomised controlled trial of a systematic care management programme aimed at improving depression scores and $\mathrm{Hb} A_{10}$, systolic blood pressure and low-density lipoprotein cholesterol levels demonstrates the cost-effectiveness of collaborative care in multimorbid conditions.

20.• Huang Y, Wei X, Wu T, Chen R, Guo A. Collaborative care for patients with depression and diabetes mellitus: a systematic review and meta-analysis. BMC Psychiatry. 2013;13:260-71. A wellwritten systematic review as well as meta-analysis of eight $R C T s$ on the treatment of diabetes and depression provides clear and conclusive evidence to support collaborative care.

21.•Watson LC, Amick HR, Gaynes BN, Brownley KA, Thaker S, Viswanaathan M, et al. Practice-based interventions addressing concomitant depression and chronic medical conditions in the primary care setting: a systematic review and meta-analysis. J Prim Care Commun Health. 2013;4(4):294-306. This is a strong systematic review and meta-analysis of the management of medical conditions in primary care and suggests areas for future research, which is important if we are to improve the knowledge and skills in collaborative care.

22. Schneider C, Jick SS, Bothner U, Meier CR. COPD and the risk of depression. Chest. 2010;137(2):341-7.

23. Hill K, Geist RR, Goldstein RSS, Lacasse Y. Anxiety and depression in end-stage COPD. Eur Respir J. 2008;31:667-77.

24. Gudmundsson G, Gislason G, Janson C, Lindberg E, Hallin R, Ulrik CS, et al. Risk factors for rehospitalisation in COPD: role of health status, anxiety and depression. Eur Respir J. 2005;26:414-9.

25. Gudmundsson G, Gislason G, Janson C, Lindberg E, Ulrik CS, Brøndum E, et al. Depression, anxiety and health status after hospitalisation for COPD: a multicentre study in the Nordic countries. Respir Med. 2006;100:87-93.

26. Unützer J, Harbin H, Schoenbaum M, Druss B. The collaborative care model: an approach for integrating physical and mental health car in Medicaid Health Homes. 2013; Center for Health Strategies and Mathematica Policy Research.

27. Collins P, Insel T, Chockalingam A, Daar A, Maddox Y (2013) Grand challenges in global mental health: integration in research, policy, and practice. PLoS Med 10(4):e1001434. doi:10.1371/ journal.pmed.1001434 http://www.plosmedicine.org/article/info\% 3Adoi\%2F10.1371\%2Fjournal.pmed.1001434

28. Wales Health and Wellbeing Best Practice and Innovation Board. The determinants of effective integration of health and social care. 2013. http://www2.nphs.wales.nhs.uk:8080/externaldocs.nsf/ 85 c 50756737 f 79 a c 80256 f 2700534 ea $3 /$
714d0d6339a19c1180257b180058a29d/\$FILE/The\% 20Determinants $\% 20$ of $\% 20$ Effective $\% 20$ Integration $\% 20$ of $\%$ 20Health\%20and\%20Social\%20Care\%20FINAL.pdf.

29.• RAND Europe, Ernst \& Young LLP (2012) Integrated Care Pilots: Final Report, Summary Version. https://www.gov.uk/government/ uploads/system/uploads/attachment_data/file/146776/dh133126. pdf.pdf. This UK report describes the challenges and opportunities for scaling up collaborative care across multiple health economies. Successful services result in a reduction in hospital admissions, hospital care and more patients cared for closer to home. The report highlights the need for economic incentives associated with patient outcomes and for patients and carers to be actively involved if collaborative care is to be successful. This document is worth reading if you are planning to set up your own integrated/ collaborative care service.

30. Dowell T, Morris C, Dood T, McLoughlin B. Psychological interventions in primary care mental health. In: Ivbijaro $\mathrm{G}$, editor. Companion to primary care mental health. London: Radcliffe Publishing; 2012. p. 241-64.

31. London Health Programmes. Mental health services case for change London. NHS London: 2010 http://www.londonhp.nhs. uk/wp-content/uploads/2011/03/1.-Case-for-change-low-res.pdf.

32. London Health Programmes. Mental health models of care for London. NHS London: $2010 \mathrm{http}: / / w w w . l o n d o n h p . n h s . u k / w p-$ content/uploads/2011/03/2.-Models-of-care-low-res.pdf.

33. Edwards TM, Švab I, Ivbijaro G, Scherger J, Clarke DD, Kellenberg GA. Multimorbidity in primary care mental health. In: Ivbijaro $\mathrm{G}$, editor. Companion to primary care mental health. London: Radcliffe Publishing; 2012. p. 672-86. This book chapter brings together the concepts of needs, risks, disability and complexity in order to stratify interventions so that people get the right care in the right place at the right time.

34. Sibbald B, Shen J, McBride A. Changing the skill-mix of the health care workforce. J Health Serv Res Policy. 2004;9(supp.1):28-38.

35. Dubois C-A, Singh D. From staff-mix to skill-mix and beyond: towards a systemic approach to health workforce management. Hum Resour Health. 2009;7:1-19.

36. Fulton BD, Scheffler RM, Sparkes SP, Auh EY, Vujicic M, Soucat A. Health workforce skill mix and task shifting in low income countries: a review of recent evidence. Hum Resour Health. 2011;9:1-11.

37. Buttorff C, Hock RS, Weiss HA, Naik S, Arya R, Kirkwood BR, et al. Economic evaluation of a task shifting intervention for common mental disorders in India. Bull World Health Organ. 2012;90: $813-21$.

38. Ivbijaro G. Aims, concept and structure of the book. In: Ivbijaro G, editor. Companion to primary care mental health. London: Radcliffe Publishing; 2012. p. 4.

39.• Taylor G, McNeill A, Girling A, Farley A, Lindson-Hawley N, Aveyard P. Change in mental health after smoking cessation: systematic review and meta-analysis. Br Med J. 2014;348:g1151. This systematic review and meta-analysis addresses cigarette smoking as one of the key links between physical and mental health morbidity. It identifies smoking cessation as a key target for improving outcomes of people with physical and mental health co-morbidity, a task that lends itself to collaborative/integrated care. Including smoking cessation programmes will result in health benefits across arrange of long-term conditions. 